\section{GRAPH: A BASIC-11 graphics program for digital plotters}

\section{WILLIAM HAYES \\ Brown University, Providence, Rhode Island 02912}

GRAPH is designed for use with the PDP-11/03 computer (Digital Equipment Corporation) and the inexpensive Hi Plot digital plotter (Houston Instrument). Figures 1 and 2 were produced in their entirety by GRAPH. The user can instruct the program to draw symbols (points or unfilled octagons, squares, diamonds, and triangles of varying size), symbols connected by lines (either solid or broken), and smooth curves (either solid or broken). Bar graphs and three-dimensional effects are possible. A title, axis labels, and tick labels are centered automatically; additional captions can be positioned at the user's discretion.

Input. This program is extremely interactive and almost completely self-explanatory. The user specifies the more important axis characteristics, such as the axis length, the scale (either linear or $\log$ ), the numerical value associated with each tick, and both a text and a character height for each label. Other axis characteristics, such as tick size and spacing, are adjusted automatically unless the override option is used.

Data can be entered in the form of $X, Y$ pairs through either the keyboard or two-dimensional virtual array BASIC files. Smooth curves are determined by up to 500 closely spaced pairs that the program causes the plotter to connect with straight lines. After a graph has been constructed, the axes can be redrawn in either identical or modified form to permit the presentation of an additional set of data.

Limitations. This program is written in BASIC-11/ RT-1 1 V2 and requires $32 \mathrm{~K}$ words of memory.

Availability. The Hi Plot digital plotter (Model DMP2) is available from Houston Instrument, One Houston Square, Austin, Texas 78753.

A listing of GRAPH and extensive documentation are available free of charge; arrangements for a copy of the program filed on floppy disk can be made by contacting the author at the Department of Psychology, Elliott Hall, University of Minnesota, Minneapolis, Minnesota 55455 .

This work was supported by United States Public Health Service Grant MH 02456 to Donald S. Blough and Patricia M. Blough. The author was supported by a Graduate Fellowship from the National Science Foundation. I would like to thank Donald Blough, Kenneth Knoblauch, and Daniel Uhlrich for their contributions at various stages in the development of this program.

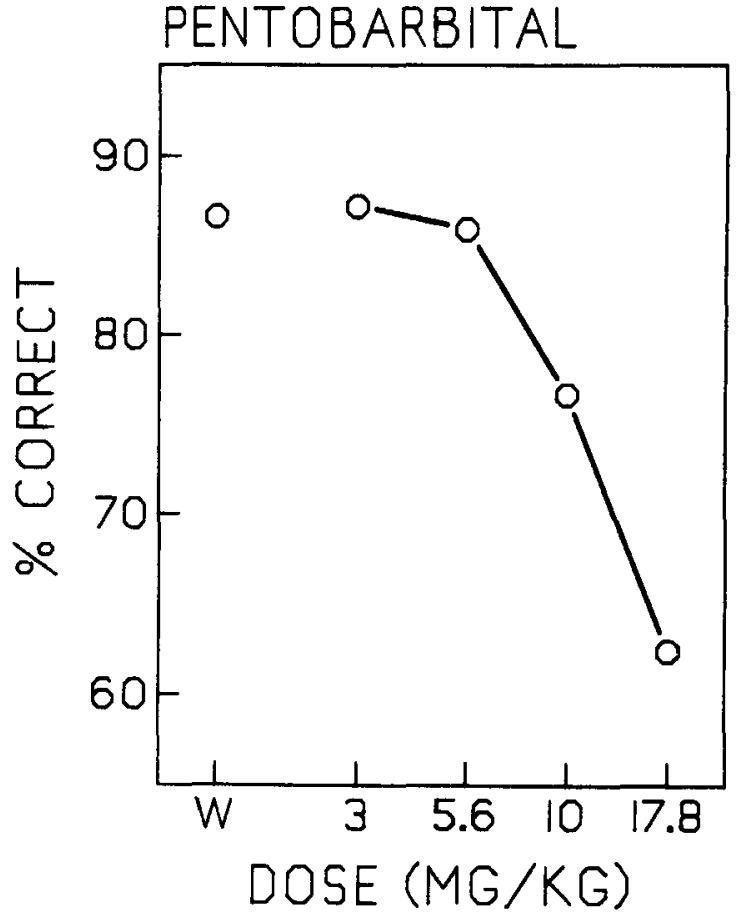

Figure 1. An example of the output produced by GRAPH.

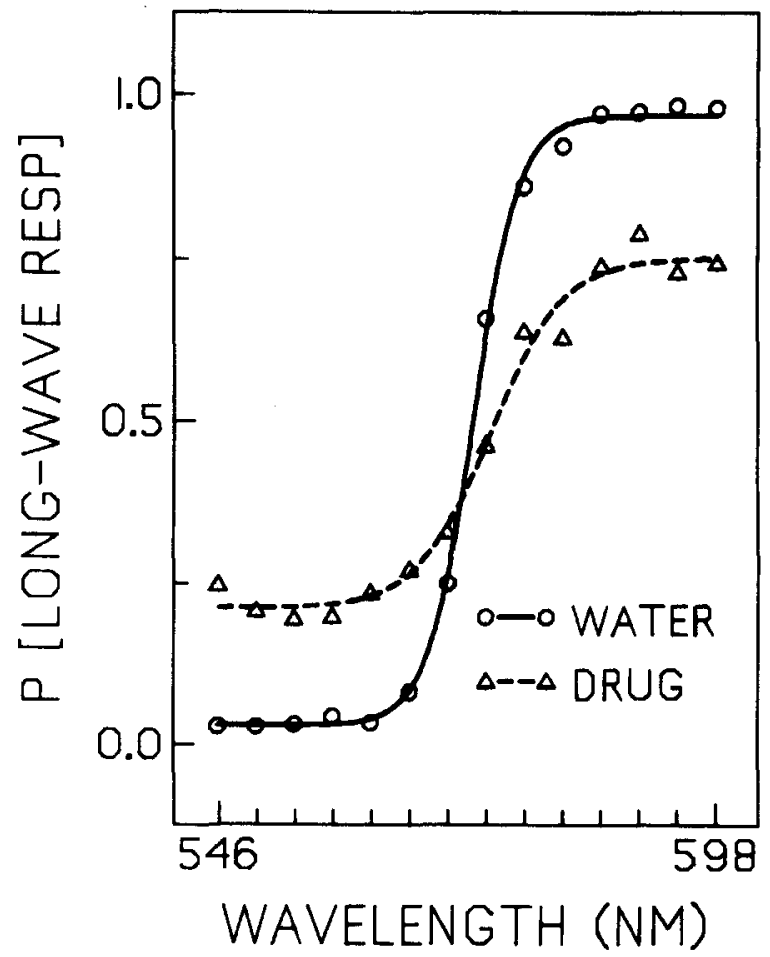

Figure 2. A second example of the output produced by GRAPH.

(Accepted for publication April 15, 1981.) 